\title{
La cultura digital en la enseñanza de la historia y las ciencias sociales. Voces del profesorado encuestado y entrevistado en una investigación
}

\author{
Digital culture in the teaching of history and social sciences. \\ Voices of teachers surveyed and interviewed in an investigation
}

Miguel Angel Jara* Agustina Tirachini**

\section{RESUMEN}

A partir de nuestra investigación nos interesa aportar a la reflexión sobre la pertinencia conceptual y metodológica y a la elaboración de estrategias de intervención en la práctica educativa. En este sentido remarcamos la importancia y potencialidad de los estudios cualitativos, que en el marco de un trabajo colaborativo y solidario entre docentes e investigadores/as, se constituye en un espacio habitado para coproducir formaciones docentes iniciales y permanentes en torno a conocimientos y prácticas del conocimiento social e histórico. Presentamos los resultados de una investigación en la que hemos construido estrategias centradas en el cuestionario y las entrevistas en profundidad como dispositivos potentes para indagaciones críticas en el campo educativo. Palabras claves: investigación cualitativa; enseñanza; cultura digital; Historia; Ciencias Sociales.

\section{Abstract}

Our research aims to discuss the development and the conceptual and methodological relevance of intervention strategies in educational practice. We emphasize the importance and potential of qualitative studies, which, as part of a collaborative and supportive work between teachers and researchers, become a living space to co-produce initial and continuing training involving practices of social and historical knowledge. We present the results of an investigation in which we have built strategies focused on questionnaire and in-depth interviews as powerful devices for critical inquiry in Education.

Keywords: qualitative research; education; digital culture; History; Social Sciences.

\footnotetext{
*Profesor en historia y Doctor en Didáctica de las Ciencias Sociales. Profesor Adjunto Regular en el área de didáctica de las Ciencias Sociales e Historia para los profesorados en nivel inicial e historia. Facultades de Ciencias de la Educación y Humanidades de la Universidad Nacional del Comahue (FACE/UNCo, FAHum/UNCo). mianjara@gmail.com

** Psicóloga. Profesora de evaluación psicológica en la carrera de psicología en la Facultad de Ciencias de la Educación de la Universidad Nacional del Comahue (FACE-UNCo). agustina tirachini@yahoo.com.ar
} 
En el proyecto de investigación, como se ha señalado en el artículo anterior de este dossier, nos hemos planteado como objetivo general analizar críticamente la red de vínculos entre medio de comunicación, nuevas tecnologías y conocimientos sociales enseñados a fin de construir dispositivos didácticos para el aprendizaje y la enseñanza de las ciencias sociales y de la historia, en instituciones educativas de los tres niveles de enseñanza en Río Negro y Neuquén.

Para ello hemos diseñado una serie de estrategias de investigación que nos han posibilitado introducirnos en la problemática como así también desandar el objeto de indagación. Dos de ellos, el cuestionario y la entrevista nos han ofrecido una serie de datos potentes para interpretar y comprender la enseñanza y el aprendizaje de las ciencias sociales y de la historia en la cultura digital. Son datos que, desde una perspectiva analítica y para nada generalizadora, nos ampliaron nuestros supuestos iniciales a la vez que nos han mostrado la complejidad de las prácticas de enseñanza de un conjunto heterogéneo de profesores y profesoras que utilizan tecnologías y soportes digitales en sus clases de historia o ciencias sociales.

A partir de nuestra investigación nos interesa aportar a la reflexión sobre la pertinencia conceptual y metodológica y a la elaboración de estrategias de intervención en la práctica educativa. Remarcamos la importancia y potencialidad de los estudios cualitativos, que en el marco de un trabajo colaborativo y solidario entre docentes terciarios/universitarios y docentes primaria/media e investigadores/as, se constituye en un espacio habitado para coproducir formaciones docentes iniciales y permanentes en torno a conocimientos y prácticas del conocimiento social e histórico.

La recolección de información obtenida del cuestionario, en la primera fase de la investigación que aquí presentamos analíticamente, ${ }^{1}$ se llevó a cabo en escuelas públicas de las provincias de Rio Negro y Neuquén. El trabajo de campo $^{2}$ se realizó sobre un total de 12 salas de nivel inicial, 12 escuelas primarias y 19 escuelas de nivel medio. La muestra la constituyen 206 mujeres y 31varones, de edades y de procedencia muy variadas. La mayoría han cursado sus estudios en Institutos de Formación Docente y en la Universidad de la región. Igualmente diversa es la antigüedad que poseen en el ejercicio de la docencia. 
Un segundo momento de recolección de información la obtuvimos a partir de entrevistas en profundidad realizadas a profesores y profesoras que accedieron al llenado del cuestionario. Hemos construido nueve casos, tres en cada nivel educativo, con los cuales iniciamos el proceso de construcción de dispositivos didácticos que serán presentados en los próximos artículos de este dossier.

Algunas de las preguntas iniciales que nos orientan en el proceso investigativo giran en torno a: ¿Qué significado de las ciencias sociales y de la historia validan estudiantes y profesores?, ¿Qué lugar le asignan a las herramientas/ máquinas en el proceso de enseñanza y de aprendizaje?, ¿Posibilitan intercambios e interacciones docentes/estudiantes más fluidas?, ¿Qué finalidades y sentidos orientan la enseñanza y el aprendizaje?, ¿Potencian la formación del pensamiento crítico y la conciencia histórica?, entre otras.

Como se planteó en el artículo de Graciela Funes - con el que se inicia este dossier - la investigación de corte cualitativo, combinado con la lógica cuantitativa, se constituyó en un proceso metodológico importante en la medida que nos ofreció un caudal de información relevante a nuestro objeto; nos habilitó la construcción de algunas categorías en las que hemos agrupado prácticas y tendencias del profesorado, vinculado a la cultura digital en la enseñanza y la construcción de casos representativos de esas prácticas y tendencias. Lo que sigue, es un análisis de datos obtenidos del cuestionario, por un lado, y el de las entrevistas en profundidad, por otro. Los presentamos de modo sintético dado la extensión del presente escrito.

\section{EL CUESTIONARIO}

Dice Bisquerra (2000), que los cuestionarios son un conjunto más o menos amplio de preguntas o cuestiones que se consideran relevantes para el rasgo, características o variables que son objetos de estudio. Distingue dos tipos de cuestionarios: los que pueden considerarse como test, que son aplicados en la medición de diagnósticos psicopedagógicos, y los de recogida de datos, que son utilizados en las encuestas.

Su elaboración no consiste en la mera enunciación de un listado de preguntas, muy por el contrario, implica considerar una cierta lógica en su organización (tipos de preguntas, grado de complejidad de las mismas, dimensiones 
o temas bien definidos y un tiempo considerable - no muy extenso - para las respuestas). Según el grado de estructuración, podemos encontrar cuestionarios estructurados, semiestructurados o no estructurados. Los primeros se caracterizan por la preeminencia de preguntas cerradas, no permite desarrollar respuestas y se presenta como un listado de opciones para señalar. El segundo, semiestructurado, es similar al primero, aunque incorpora algunas preguntas abiertas y da la posibilidad, al encuestado, de elaborar las respuestas. Los últimos, se caracterizan por la existencia de preguntas abiertas, en las que se elaboran las respuestas. Generalmente son utilizados en las entrevistas en profundidad. En nuestras investigaciones hemos construido cuestionarios semiestructurados y su organización ha reflejado los objetivos de la pesquisa.

El cuestionario puede considerarse como una entrevista formalizada y estilizada, o una especie de sustituto de ésta. Formalmente es lo mismo que una entrevista cara a cara, aunque a fin de prescindir de la presencia del entrevistador/a, se presenta al sujeto lo que podría definirse como una transcripción estructurada de entrevista sin respuesta. El cuestionario es una especie de entrevista en serie y, como tal, presenta algunos de los problemas típicos de la producción en masa, sobre todo en lo que respecta a la falta de oportunidades de interpretación. Por otra parte, ofrece numerosas ventajas de cara a su administración, al presentar, al menos potencialmente, un estímulo idéntico a numerosos sujetos de forma simultánea, proporcionando al investigador la oportunidad de acumular datos con (relativa) facilidad (Walker, 1989, p.114).

Por lo general, dice Walker (1989, p.130), los cuestionarios son aplicables a muestras numerosas de sujetos y plantean preguntas bastantes superficiales, o, al menos, preguntas cuidadosamente adaptadas a respuestas predeterminadas. También pueden emplearse en situaciones más localizadas e íntimas. Este enfoque del diseño de cuestionarios ofrece una forma de integrar datos "cualitativos" y "cuantitativos", aunque hay que señalar que Stake y Easley encontraron que esta integración era más difícil de conseguir de lo que esperaban. En primer lugar, resultaba difícil hallar escenarios apropiados a partir del estudio de casos: siempre había que retocarlos para obtener los ítems del cuestionario. En segundo lugar, al analizar los datos resultantes, las respuestas a los cuestionarios no encajaban bien con el material de los estudios de casos, o al menos no proporcionaban una forma directa de corroborarlos o refutarlos como cabía esperar. 
En este sentido y considerando los recaudos metodológicos, en la primera fase de esta investigación, hemos utilizado un cuestionario que nos permitió construir los datos, que luego analizaremos, a partir de cuatro bloques: I) datos personales, II) situación académica, III) situación laboral y IV) práctica de la enseñanza.

Las preguntas que le dieron cuerpo a cada uno de los bloques, (fundamentalmente en el III y IV) nos permitieron conocer a los/as profesores/as, las instituciones en las que desarrollan su trabajo y aspectos centrales de su práctica de la enseñanza con la utilización de las nuevas tecnologías. En el bloque III, las preguntas se centraron en las características de las instituciones en las que trabajan, en cuanto a la infraestructura y soportes tecnológicos existentes, planes o programas educativos y la utilización de estos en sus tareas docentes. En el último bloque preguntamos sobre las nuevas tecnologías, conocimientos sobre ellas y grado de utilización en las prácticas de enseñanza o en la vida cotidiana del profesorado. Combinamos preguntas abiertas con preguntas cerradas. En varias de ellas se les presentó una serie de opciones que debían valorar y, en algunas, se les solicitó que fundamentaran o desarrollaran brevemente sus respuestas. En general todo el instrumento se diseñó en función de los objetivos de la investigación y resultó pertinente a esta primera fase.

La construcción del cuestionario no fue azarosa, procuramos organizar en cada bloque un conjunto de preguntas que nos posibilitaron tener una primera mirada sobre la existencia, acceso y usos de las nuevas tecnologías en la enseñanza. De la lectura y construcción de los datos, a partir de la información recogida con el instrumento, obtuvimos un primer panorama general sobre la existencia de prácticas docentes, diversas y para nada homogéneas, que nos ampliaron ciertos supuestos iniciales y que serán profundizados en las entrevistas individuales. El instrumento construido en esta primera fase, en cada una de sus dimensiones, nos aportó un conjunto de datos que nos habilita a un primer análisis y que, posteriormente, serán triangulados, junto a los datos recabados de las entrevistas, planificaciones, secuencias didácticas y registros de observación de clases, con la finalidad de construir un conocimiento didáctico.

\section{¿QUÉ NOS DICEN LOS DATOS?}

La organización del cuestionario, en el marco de una investigación cualitativa critica como la que llevamos a cabo, nos permitió adentrarnos a las 
prácticas y perspectivas del profesorado y desentrañar la complejidad de concepciones y usos de las nuevas tecnologías en la enseñanza. Entendemos que esa complejidad no nos habilita a establecer generalidades, muy por el contrario, el análisis de los datos que presentamos atienden a casos particulares de nuestra muestra y pretenden dar cuenta de las significaciones del profesorado en cuanto al universo de posibilidades que le plantean las nuevas tecnologías a los procesos de enseñanza y de aprendizaje de las ciencias sociales y de la historia en la cultura digital.

En el proceso investigativo, los/as actores/as (profesorado/estudiantes/ investigador) interactúan en un marco de sentidos, experiencias y prácticas diversas. Por ello, en el análisis, hemos procurado realizar una comprensión e interpretación de los significados que subyacen en lo "dicho" por los/as encuestados/as de manera que nos posibiliten desandar sus referencias y concepciones, en un espacio y tiempo particular.

Como hemos anticipado más arriba, la muestra fue aleatoria e intencional. De la totalidad de personas encuestadas (ver Gráfico 1) el 87\% son mujeres de entre 20 y más de 50 años de edad y solo un $13 \%$ son varones de entre 20 a 50 años de edad (ver Gráfico 2). El criterio de agrupamiento etario por décadas se justifica en la diversidad de edades de los/as encuestados/as, a la edad cronológica de inserción a los estudios de grado e incorporación al mundo del trabajo en las instituciones educativas. Finalmente, el criterio etario nos posibilita identificar generaciones de profesores/as y sus rasgos identitarios y culturales (Margulis; Urresti, 2008).

La mayoría de los varones de la muestra son profesores en historia en la escuela media. Nos encontramos con una población de profesores/as que desarrollan su trabajo en contextos socio comunitario e institucionales diversos y han realizado sus estudios de grado (maestros/as o profesores/as de las disciplinas) entre las décadas de los 1990 y 2000. En su mayoría son profesores/ as con una importante antigüedad en la docencia (46\% posee más de 11 años de antigüedad en la docencia - ver Gráfico 3).

Estos datos nos permiten hacer una primera lectura que articula contextos, formación y experiencias. Sabemos que los/as profesores/as cursaron sus estudios de grados en un contexto particular de la historia de nuestro país y del mundo (ver Gráfico 4). Durante los años 1990 el 25\% de los/as docentes encuestados obtuvieron su título docente. Fueron años de avance y 

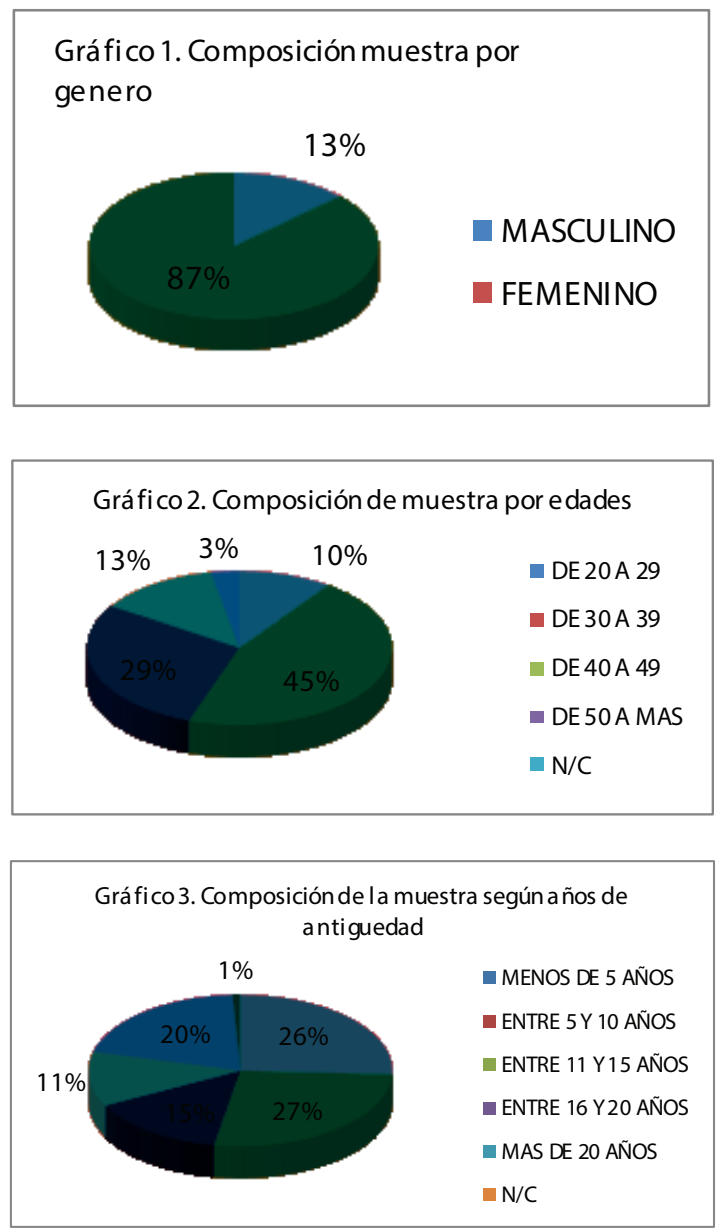

profundización de políticas neoliberales que implicaron reformas estructurales en diversos aspectos de la sociedad y el estado. En el plano educativo significó un corrimiento de la principalidad del estado en materia educativa, de garantizar condiciones de trabajo y de infraestructura, entre otras.

Las sucesivas reformas curriculares para la educación obligatoria, ligadas a la demanda del mercado, no fueron acompañadas de programas de formación inicial y continuada del profesorado y, en algunos casos, hubo resistencia al avance de estos programas en universidades e institutos de formación 
docente. Fue una década inaugural y vertiginosa en cuantos a los cambios sociales, económicos, políticos y tecnológicos, sin precedentes en la reciente historia de nuestro país. Cambios que imponen nuevas racionalidades, desde las definiciones políticas del estado, que no son acompañadas por la "logística" que demanda su implementación. Se produce una especie de enmascaramiento de la realidad sostenida solo desde lo discursivo. El avance desenfrenado de la lógica neoliberal provocó más desagregación, marginalidad y exclusión de lo que se sostenía del discurso mesiánico de la salvación social. Situación que se manifestó durante la década que inaugura el nuevo milenio en diversidad de conflictos sociales y educativos a lo largo y ancho de la geografía de nuestro país; década en la que la mayoría de nuestra muestra (el 33\%) finalizó sus estudios docentes. En esta línea podríamos continuar el análisis, pero a los efectos de este trabajo, procuraremos establecer las relaciones de los contextos con la formación.

Las reformas y contrarreformas ${ }^{3}$ experimentadas en el sistema educativo de la provincia de Rio Negro (en los tres niveles educativos) incorporaron la enseñanza en tecnologías de forma irregular en la jurisdicción. Existieron pocos casos de escuelas primarias (fundamentalmente privadas o públicas de gestión privadas) que incorporaron a las nuevas tecnologías como espacio curricular. Para el nivel medio solo se dio en las escuelas que ofrecían esa modalidad u orientación en el bachillerato o ciclo superior y era una asignatura más que no tenía mayor vinculación con otros espacios curriculares. En las escuelas públicas de la provincia de Neuquén existen pocas experiencias, generalmente promovidas por las mismas instituciones, que han incorporado computación, informática o tecnología como espacio curricular para las orientaciones en comunicación o tecnologías. El sistema educativo provincial se caracteriza por la ausencia de un diseño curricular para el nivel medio y la existencia de diversos planes de estudios, que en algunos casos poseen más de 50 años.

En ambas provincias, la última década se ha caracterizado por marchas y contramarchas en la construcción de un currículo de alcance provincial, para la educación media. En el caso de Rio Negro, la reciente reforma plantea la importancia de la tecnología de la información en la enseñanza, cuestión que se evidencia fuertemente en los argumentos y finalidades de la enseñanza y el aprendizaje en los actuales contextos, pero, paradójicamente esta 
intencionalidad no se condice con los contenidos propuestos para el área de las ciencias sociales, por ejemplo. Neuquén, al momento, no ha logrado concretar una reforma participativa, carece de un diseño curricular aunque ha comenzado el proceso de construcción para implementarlo, en todo el territorio provincial, durante el año 2016.

Para el caso de la formación del profesorado esta situación no se constata en el ámbito universitario. Recientemente los Institutos de Formación Docente, previa reforma e implementación del nuevo plan de estudios (año 2008/2011), han incorporado este espacio en la formación, en ambas provincias. En este periodo se ubica un $17 \%$ de profesores/as encuestado, como lo indica el Gráfico 4.

No se desconoce, en estas últimas tres décadas por lo menos, que han habido iniciativas y políticas concretas desde los estados (nacional y provincial) de "modernizar" a la escuela con la nueva tecnología. Innovación que requiere de una fuerte inversión y que, paradójicamente, no fue considerado masivamente para la escuela pública, al menos en los años 1990. En los últimos años se ha desarrollado un programa de equipamiento de netbooks, de forma masiva y de una importante inversión, a cierto nivel educativo. Sin embargo, como sostiene Inés Dussel (2010, p.38) “el equipamiento es un factor imprescindible aunque insuficiente, si no se lo combina con una definición del para qué y el cómo se introducen las nuevas tecnologías".

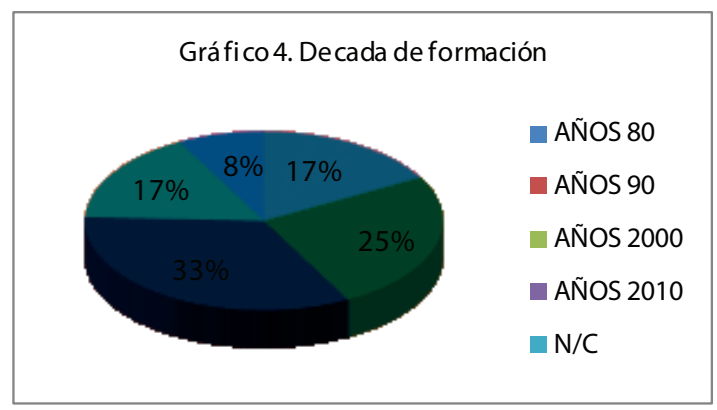

Entonces, considerar los contextos de formación, de construcciones curriculares y de ejercicio de la docencia es un dato relevante para comprender como estas generaciones de profesores/as se vinculan con las nuevas tecnologías y cuáles son los usos que de ellas hacen en sus clases. Los procesos de 
construcción de nuevas racionalidades en la formación del profesorado son, a nuestro entender, el punto de partida para comprender la tradición o los cambios y/o innovaciones en la enseñanza.

Aun cuando el avance y desarrollo vertiginoso y masivo de las nuevas tecnologías impacta fuertemente en la vida cotidiana de las personas, no sucede lo mismo en el sistema educativo. La existencia de la nueva tecnología en la era digital, no se ha incorporado ni convertido en materia de enseñanza y de aprendizaje en los tres niveles educativos, ni en el sistema formador del profesorado universitario. Una situación que pone al desnudo los límites y posibilidades de su incorporación a la enseñanza.

Los datos obtenidos del cuestionario indican que, del total de la muestra (237 personas), el 61\% han adquirido conocimiento en nuevas tecnologías para la enseñanza, de forma autodidacta (Ver Gráfico 5). Los datos nos indican, también, que el potencial y predisposición que presenta el profesorado sería una buena oportunidad para ofrecer formación específica en las nuevas tecnologías, de forma continuada a través de un plan de estudios que supere los límites políticos de un programa o proyecto.

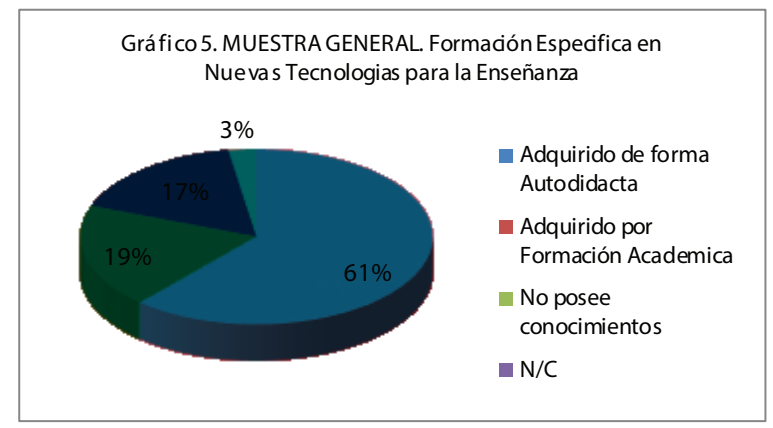

Al profundizar en las características particulares de cada nivel, se observan ciertas similitudes en cuanto a su prevalencia por sobre la formación académica. Para el caso del nivel inicial el 75\% sostiene que su conocimiento fue adquirido de manera autodidacta, mientras que el 13\% reconoce haberse formado a partir de instancias académicas, porcentaje significativamente similar al 12\% que manifiesta no poseer conocimientos específicos.

Para el caso de nivel primario, los resultados obtenidos indican que un $50 \%$ se ha formado de manera autodidacta, el $24 \%$ ha recibido formación 
académica y un $21 \%$ expresa no tenerlos. Mayor es el porcentaje (69\%) de autodidactas para el nivel medio, mientras que solo un 17\% recibió formación académica y el resto, $14 \%$, no posee conocimiento específico.

La relevancia de la información nos indica que el 61\% de la muestra no ha recibido formación académica en el uso de las nuevas tecnologías y que sus conocimientos se han producido de manera autodidacta. Ello, conjeturalmente, nos permite sostener que el conocimiento que se posee es un conocimiento práctico que se ha adquirido por la mecánica que indica el ingreso y navegación a internet o las orientaciones ofrecidas por el software o programas utilizados. De esta manera nos encontramos frente a una realidad que pone el énfasis en una racionalidad técnica y operativa en el uso de la maquina/herramienta; y esto, a nuestro entender, es un límite sino se trabaja en la formación específica y el valor del conocimiento que se produce en los contextos digitales, de internet y de programas que circulan en la web.

A partir del año 2010, por políticas promovidas desde el estado nacional, se creó el programa Conectar Igualdad ${ }^{4}$ con la finalidad de reducir la brecha digital en la escuela pública. Es un programa que tiene como finalidad distribuir netbooks a estudiantes y docentes de escuelas secundarias, de educación especial e institutos de formación docente. Entre otros objetivos, según el programa, se plantea la alfabetización en las TIC y generar profundas transformaciones para fortalecer una nueva escuela en la Argentina.

En este marco, una cuestión no menor, es considerar las características de infraestructura de las instituciones educativas de la muestra. Existe diversidad de situaciones edilicias, de soporte técnico y de infraestructura específica para la incorporación, el uso y acceso a la nueva tecnología para la enseñanza, según podemos ver en el Gráfico 6. Si desagregamos estos datos por niveles del sistema educativo nos encontramos con lo siguiente: en la escuela media - que está contemplada por el programa nacional - solo el $40 \%$ posee servidor de internet, el $20 \% w i$-fi, el $40 \%$ posee laboratorios o salas de informática y un $3 \%$ cuenta con gabinetes móviles. Datos que no varían mucho para los otros niveles del sistema educativo: Nivel Inicial (Servidor de internet 43\%, wi-fi 22\%, laboratorio o sala de informática, 6\%, gabinetes móviles). Nivel Primario (Servidor de internet $32 \%$, wi-fi $31 \%$, laboratorio o sala de informática $20 \%$, gabinetes móviles 7\%). 


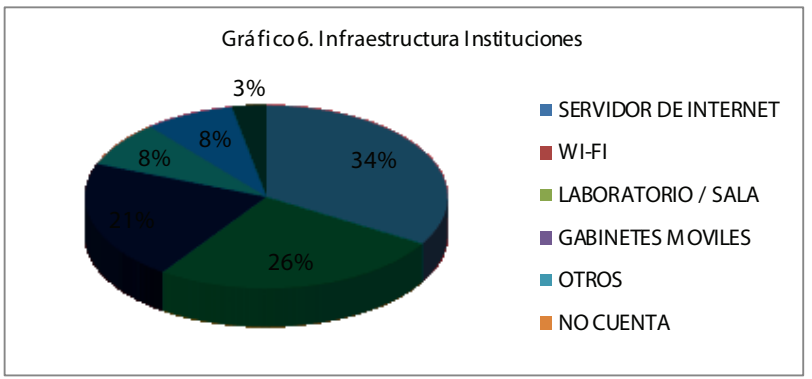

Decimos que no es menor el dato de la infraestructura y soportes tecnológicos que poseen las escuelas en tanto ello presupondría un impacto o transferencia en la enseñanza. Lo evidente es que, más allá de la existencia de un programa nacional y/o jurisdiccional que promueven la incorporación de las TIC en la enseñanza se debe considerar la presencia de la infraestructura que facilite su utilización. La existencia de estos recursos e infraestructura, según nuestra muestra, no son escasas, si tomamos como referencia el total de los datos obtenidos. Un $64 \%$ de los y las docentes encuestados nos confirman que sí utilizan la infraestructura disponible; mientras que aquellos que no lo hacen (33\%) justifican sus respuestas por las siguientes causas: $21 \%$ por mal funcionamiento, $28 \%$ por desconocimiento, $20 \%$ por falta de personal idóneo, el $16 \%$ por falta de actualización y mantenimiento y un 15\% NC.

Si bien estos datos son significativos, no estaríamos en condiciones de asegurar que ello presupone un cambio en las prácticas de enseñanza. Como sostiene Dussel (2010) la incorporación de soportes tecnológicos e infraestructura no garantiza su uso, en tanto consideramos que ello implica conocimiento específico. Citando a Cuban (2008), la autora sostiene que existe "la creencia que la presencia de los aparatos producirá per se otra relación con el conocimiento y se sobreestima la capacidad de transformación de los aparatos por sobre sus condiciones de recepción, apropiación y modos de uso" (Dussel, 2010, p.156).

Cuando preguntamos específicamente si utilizan las nuevas tecnologías en la enseñanza de las ciencias sociales e historia, las respuestas son igualmente interesantes, el $70 \%$ sostiene que si las utiliza frente a un $26 \%$ que no lo hace por desconocimiento o disponibilidad entre otras causas. En cuanto a los y las docentes que si las utilizan como recursos didácticos prevalecen las herramientas 
audiovisuales y el uso de la PC. También dicen consultar páginas web, programas oficiales, buscadores, enciclopedias digitales, etc.

\section{LAS ENTREVISTAS}

La entrevista en profundidad ha sido el otro instrumento que hemos utilizado en la investigación y que, a diferencia del cuestionario semiestructurado, nos proporcionó información oral, en una relación interpersonal, en un acto conversacional con el profesorado. A aquellas preguntas iniciales que orientaron la construcción de nuestro diseño de investigación, planteadas más arriba, se incorporaron otras preguntas que nos sugirieron de los datos del cuestionario ya sistematizado, de manera que enriquecieron el dialogo con nuestros/ as entrevistados/as.

Las entrevistas realizadas, correspondiente a la segunda fase de nuestro proceso investigativo, fueron seleccionadas entre la población total encuestada. El criterio fue la disposición del profesorado a ser entrevistado; con la idea de que se constituyan en casos con los que trabajaríamos en la construcción de dispositivos didácticos. Realizamos entrevistas antes de comenzar con el trabajo colaborativo en la construcción del dispositivo y al finalizar la implementación del mismo.

Nos hemos guiado por una serie de temas, con el objetivo de dialogar sobre cuestiones relevantes a nuestro proyecto, la conversación giró en torno a lo siguiente:

a) Ingreso a la docencia,

b) planificación de la enseñanza y secuencias didácticas (criterios de organización de los contenidos, material de enseñanza),

c) el trabajo y la explicación en el aula de historia,

d) incorporación de la cultura digital en sus propuestas y

e) opiniones y experiencias sobre los límites y las posibilidades de la enseñanza y el aprendizaje a partir de la utilización de la nueva tecnología y los recursos digitales. 
¿Por qué creemos en la efectividad de la entrevista respecto de esta investigación? A partir de esta pregunta, trataremos la delimitación metodológica de la entrevista y los desafíos que nos plantea. En el marco de los objetivos de esta investigación no deja de ser interesante poder "objetivar" nuestra mirada una y otra vez, a través de la entrevista. Teniendo presente que nos permite dar cuenta no sólo de hechos y comentarios sino también de percepciones y "descuidos", de la combinación de visión y no visión. En última instancia, pero no menos importante, descubrir respuestas a preguntas no siempre formuladas.

Dice Walker (1989, p.114) que la entrevista es un método o conjunto de técnicas específicas de las ciencias sociales y humanas. Abarca una amplia gama de técnicas, desde los cuestionarios estructurados, hasta la conversación "no estructurada", todas las cuales se basan en el supuesto de que las personas pueden reflexionar, hasta cierto punto, sobre sus propias acciones o, al menos, se les puede inducir a hacerlo. Este rasgo de la entrevista la convierte en un instrumento preferido por los defensores del "movimiento" de profesores investigadores, ya que admite cierto margen de autoridad a los sujetos y da por sentado que las explicaciones de éstos tienen un valor de verdad.

En el caso particular de las entrevistas realizadas hemos sido cuidadosos, porque entre entrevistado-entrevistador se comparte una disciplina, un marco similar de referencia, diferenciado por los fundamentos propios sobre la enseñanza y el aprendizaje, y cuestiones éticas e ideológicas. Se trata de una entrevista donde se comparten problemas comunes, donde aparecen ventajas, así como también posibles conflictos que afectan la interpretación. Tratamos, en el transcurso de la entrevista, de entablar una comunicación sin presiones, sin la sensación de examen ni que se pone en tela de juicio sus conocimientos, capacidades o habilidades. Es una cuestión que consideramos muy importante para el desarrollo de la entrevista (Jara; Santisteban, 2010).

La entrevista posibilita la apertura a un universo subjetivo, discursivo permite indagar las percepciones y las prácticas que "otro/a" realiza -, sobre las cuales no se trata sólo de verificar lo que el/la investigador/a cree de antemano o de refutarlo, sino que permite conocer, comprender, explicar y por ende enriquecer su propia formación y su propio campo de trabajo. Gracias a su potencialidad discursiva, a la capacidad de ejemplificación, simbolización y concreción, la entrevista puede ejercer un efecto de revelación. Esto debido a que también permite entregar un equivalente más accesible de análisis 
conceptuales complejos, haciendo sensibles las estructuras objetivas que el trabajo científico se esfuerza por destacar.

Como acto conversacional, ${ }^{5}$ la entrevista es una técnica importantísima para la recolección de información, en función de los propósitos que diseñe el/la investigador/a. Existen diversos tipos de entrevista, que pueden ser clasificadas por su grado de estructuración o bien por la presencia o no del entrevistado. Entre las primeras se reconocen las estructuradas, semi-estructuradas y no estructuradas (Burguess, 1984). Para la segunda de las clasificaciones se establece una distinción del acto conversacional que varía entre el intercambio directo (cara a cara) y el indirecto (verbal telefónico) (Fideli; Marradi, 1996). En nuestro caso hemos optado por un intercambio directo.

Como bien señala Piovani (2007) la esencia de este tipo de entrevista es su destacado nivel de subjetividad, habida cuenta que será el/la entrevistador/a quien le dará la significación y la pertinencia que merece. Es quizás en este punto que subyace su limitación más importante; es decir, que en este acto conversacional la potencialidad y riqueza del mismo depende de las capacidades, experticias y personalidad del entrevistador/a. Sin embargo, también se reconoce una ventaja muy importante para este tipo de entrevistas en tanto que "permite obtener de manera flexible una información rica y profunda, en las propias palabras de los actores" (Valles, 1997, p.196-198). La relación, entonces, entre las ventajas y desventajas, radicarían en el grado de empatía que se establezca entre el/la entrevistado/a y el/la entrevistador/a, en función de la potencialidad de la información obtenida. La elección de temas para la entrevista posibilitará la construcción de un determinado discurso, una narración, un relato, como una descripción espontanea que hace el/la informante sobre sus propias acciones, experiencias, sentidos y creencias de sus recuerdos. Por ello los datos provenientes del entrevistado/a conforman una importante fuente de información.

La clave de la entrevista consiste en el diseño de una guía más o menos estructurada que varía, según el grado de familiaridad que tenga el/la investigador/a con el tema en cuestión. Según Valles (1997, p.219), se trata de "trazar un esquema en el que se anticipen los modos de abordar el tema central y las cuestiones secundarias ... supone tener listas preguntas de amplio espectro para los inicios; así como una serie de cuestiones y argumentos que sirvan 
para pasar de unos asuntos a otros; o para motivar al entrevistado" (citado en Marradi; Archenti; Piovani, 2007, p.223). Sin embargo, esta guía no debe operar como un instrumento de interrogación sino más bien como una ayuda memoria para no olvidar los temas relevantes para la investigación. Tampoco debería priorizar o establecer orden en el acto conversacional.

Otra de las cuestiones igualmente importantes es la que refiere a los criterios de selección de los informantes claves (muestreo intencional y oportunista). Si bien para algunos acontecimientos no resulta "difícil" encontrar colaboradores, esto no siempre sucede. "Cuando se trata de personas que por sus características no son fácilmente identificables por parte del investigador se suele recurrir a la técnica de bola de nieve (snowball), a partir de uno o unos pocos contactos iniciales, y valiéndose de las redes personales de los mismos, se busca ampliar progresivamente el grupo de potenciales entrevistados que compartan aquellas características que los hacen minoritarios y poco visibles y accesibles" (Marradi; Archenti; Piovani, 2007, p.223).

\section{¿QUÉ NOS DICEN LOS PROFESORES Y LAS PROFESORAS?}

Lo que presentamos en este apartado son las experiencias de algunos/as de nuestros/as entrevistados/as. A modo de ejemplo hemos construido un cuadro en el que sintéticamente volcamos expresiones que son representativas de los decires del profesorado entrevistado. Hemos optado por esta modalidad para presentar al lector/a algunas dimensiones que nos permiten comparar los tres niveles obligatorios del sistema educativo argentino. Las categorías de agrupamiento comparativo devienen de la conversación con el profesorado y dan cuenta de las valoraciones, tendencias y prácticas en la enseñanza de las ciencias sociales e historia.

De un primer análisis de las entrevistas realizadas, consideramos importante tener en cuenta algunas cuestiones relacionadas al recorrido que ha transitado la construcción y trasmisión del conocimiento histórico y los modos de comunicación de la información y del conocimiento que la escuela tiene como desafío. 


\begin{tabular}{|c|c|c|c|}
\hline $\begin{array}{l}\text { Dimensiones } \\
\text { Comparativas }\end{array}$ & $\begin{array}{l}\text { Nivel Inicial } \\
\text { (salitas de } 4 \text { y } 5 \\
\text { años de edad) }\end{array}$ & $\begin{array}{l}\text { Nivel Primario } \\
\text { (6 a } 12 \text { años } \\
\text { de edad) }\end{array}$ & $\begin{array}{l}\text { Nivel Medio } \\
\text { (13 a } 18 \text { años } \\
\text { de edad) }\end{array}$ \\
\hline $\begin{array}{l}\text { Proyecto } \\
\text { Institucional } \\
(\mathrm{PI})\end{array}$ & $\begin{array}{l}\text { "En el año } 2012 \text { nos } \\
\text { hacen entrega de PC, } \\
\text { en el marco del } \\
\text { Programa Aula } \\
\text { Virtual y a partir de } \\
\text { ahí desarrollamos un } \\
\text { proyecto institucional } \\
\text { para justificar el uso } \\
\text { del recurso y la } \\
\text { implementación de las } \\
\text { nuevas tecnologías en } \\
\text { las salitas de } 4 \text { y } 5 \text { " (se } \\
\text { refieren a la edad de } \\
\text { los niños y las niñas) }\end{array}$ & $\begin{array}{l}\text { "la escuela cuenta } \\
\text { con un PI que } \\
\text { incorpora el } \\
\text { programa de la sala } \\
\text { primaria digital en } \\
\text { la enseñanza" }\end{array}$ & $\begin{array}{l}\text { "La institución fue } \\
\text { incorporada al } \\
\text { Programa Nacional } \\
\text { Conectar Igualdad" }\end{array}$ \\
\hline $\begin{array}{l}\text { Formación } \\
\text { específica } \\
\text { en TICS }\end{array}$ & $\begin{array}{l}\text { Predomina la } \\
\text { capacitación técnica a } \\
\text { fin de comprender } \\
\text { como hacer uso del } \\
\text { recurso/soporte. "el } \\
\text { primer año cuando } \\
\text { nos llegó el aula } \\
\text { virtual tuvimos dos } \\
\text { encuentros con el } \\
\text { referente TICs donde } \\
\text { vimos el uso del } \\
\text { proyector y de las } \\
\text { maquinas" } \\
\text { En general } \\
\text { manifiestan la } \\
\text { necesidad de } \\
\text { formación en TICs } \\
\text { para su incorporación } \\
\text { en los procesos de } \\
\text { enseñanza y de } \\
\text { aprendizaje específica } \\
\text { para el nivel inicial. }\end{array}$ & $\begin{array}{l}\text { Pocas docentes } \\
\text { tienen } \\
\text { conocimiento } \\
\text { operativo de las } \\
\text { TICs. Cuentan con } \\
\text { un Referente } \\
\text { Pedagógico (RP) } \\
\text { quien orienta y } \\
\text { ayuda en el } \\
\text { conocimiento } \\
\text { específico de las } \\
\text { nuevas tecnologías } \\
\text { (NT). }\end{array}$ & $\begin{array}{l}\text { En general son pocas } \\
\text { las/los docentes que } \\
\text { poseen conocimiento } \\
\text { para la incorporación } \\
\text { de las TICs en los } \\
\text { procesos de } \\
\text { enseñanza y de } \\
\text { aprendizaje. La } \\
\text { implementación y } \\
\text { uso de las nuevas } \\
\text { tecnologías queda } \\
\text { supeditado al interés } \\
\text { del docente y su } \\
\text { capacidad. "Es una } \\
\text { tarea que demanda } \\
\text { tiempo si no querés } \\
\text { quedarte solo con lo } \\
\text { que tiene la plantilla } \\
\text { de Conectar } \\
\text { Igualdad" }\end{array}$ \\
\hline
\end{tabular}




\begin{tabular}{|c|c|c|c|}
\hline $\begin{array}{l}\text { Recursos y } \\
\text { soportes } \\
\text { tecnológicos/ } \\
\text { digitales }\end{array}$ & $\begin{array}{l}\text { Cuentan con } \\
\text { netbooks del } \\
\text { programa Aula } \\
\text { Virtual, también con } \\
\text { proyector, } \\
\text { micrófonos y } \\
\text { auriculares. } \\
\text { Disponen de un } \\
\text { software educativo } \\
\text { diseñado } \\
\text { específicamente para } \\
\text { el nivel inicial. } \\
\text { Han incorporado el } \\
\text { uso de internet } \\
\text { (domiciliario) para } \\
\text { consultar páginas } \\
\text { web en busca de } \\
\text { contenidos para } \\
\text { llevar al aula. } \\
\text { Predominan entre } \\
\text { ellas Pakapaka, canal } \\
\text { encuentro, Unicef, } \\
\text { etc. }\end{array}$ & $\begin{array}{l}\text { Poseen un } \\
\text { programa } \\
\text { educativo: “Aviones } \\
\text { de Papel” diseñado } \\
\text { por el R.P. } \\
\text { Consultan páginas } \\
\text { web como por } \\
\text { ejemplo: Educ.ar, } \\
\text { Canal Encuentro, } \\
\text { Pakapaka. } \\
\text { Cuentan con los } \\
\text { recursos } \\
\text { proporcionados por } \\
\text { el Programa } \\
\text { Primaria Digital y } \\
\text { otros recursos } \\
\text { tecnológicos } \\
\text { adquiridos por la } \\
\text { escuela }\end{array}$ & $\begin{array}{l}\text { Cuentan con los } \\
\text { recursos } \\
\text { proporcionados por } \\
\text { el Programa } \\
\text { "conectar Igualdad" } \\
\text { como las Netbooks } \\
\text { para docentes y } \\
\text { estudiantes. También } \\
\text { con otros recursos } \\
\text { tecnológicos } \\
\text { adquiridos por la } \\
\text { escuela (Ej. } \\
\text { proyector). }\end{array}$ \\
\hline $\begin{array}{l}\text { Modos de } \\
\text { utilización de } \\
\text { las nuevas } \\
\text { tecnológicas } \\
\text { (NT) }\end{array}$ & $\begin{array}{l}\text { Se evidencian tres } \\
\text { modalidades } \\
\text { diferentes y } \\
\text { completarías en el } \\
\text { uso de las NT. } \\
\text { Mayormente utilizan } \\
\text { las NT como soporte } \\
\text { didáctico de manera } \\
\text { complementaria a los } \\
\text { soportes } \\
\text { tradicionales. El uso, } \\
\text { en este caso, esta } \\
\text { mayoritariamente } \\
\text { dado por la material, } \\
\text { y no por la creación } \\
\text { del mismo. }\end{array}$ & $\begin{array}{l}\text { El software libre } \\
\text { diseñado por el RP, } \\
\text { les permite a las } \\
\text { docentes construir } \\
\text { actividades, junto a } \\
\text { los niños/as, para } \\
\text { abordar } \\
\text { determinados } \\
\text { contenidos. Ej. } \\
\text { Juegos, libros } \\
\text { digitales, sopa de } \\
\text { letras, videos. } \\
\text { Utilizan el } \\
\text { programa Aviones } \\
\text { de papel, } \\
\text { fundamentalmente }\end{array}$ & $\begin{array}{l}\text { Se utilizan las NT } \\
\text { para la planificación } \\
\text { de clases, el } \\
\text { desarrollo de } \\
\text { contenidos a partir } \\
\text { de la construcción de } \\
\text { redes y mapas } \\
\text { conceptuales, se } \\
\text { escanean textos } \\
\text { varios y partes de } \\
\text { libros, que les son } \\
\text { útiles para trabajarlos } \\
\text { con los/as } \\
\text { estudiantes. } \\
\text { Se realizan } \\
\text { PowerPoint, que } \\
\text { luego son }\end{array}$ \\
\hline
\end{tabular}




\begin{tabular}{|c|c|c|c|}
\hline & $\begin{array}{l}\text { Así también, se hace } \\
\text { un uso frecuente de } \\
\text { (video) juegos } \\
\text { articulados a los } \\
\text { contenidos } \\
\text { planificados. Actúan } \\
\text { como recurso } \\
\text { movilizador y no } \\
\text { como un mero } \\
\text { entretenimiento. } \\
\text { En menor medida, } \\
\text { han podido } \\
\text { implementar } \\
\text { secuencias didácticas } \\
\text { que fomentan la } \\
\text { producción propia de } \\
\text { nuevos contenidos, a } \\
\text { partir del material } \\
\text { producido en la } \\
\text { participación activa } \\
\text { conjunta de los niños } \\
\text { y la docente; } \\
\text { otorgando un nivel } \\
\text { mayor de } \\
\text { significación al } \\
\text { propio recurso. }\end{array}$ & $\begin{array}{l}\text { para trabajar } \\
\text { geografía }\end{array}$ & $\begin{array}{l}\text { proyectados en un } \\
\text { cañón, al igual que } \\
\text { los cortos y películas } \\
\text { que se utilizan para la } \\
\text { explicación de ciertos } \\
\text { contenidos. } \\
\text { Hacen uso de juegos } \\
\text { en red para abordar } \\
\text { temáticas específicas } \\
\text { como por ejemplo la } \\
\text { Revolución Francesa. } \\
\text { También utilizan } \\
\text { crucigramas y } \\
\text { anagramas digitales. }\end{array}$ \\
\hline $\begin{array}{l}\text { Valoración } \\
\text { profesoral de las } \\
\text { NT en la } \\
\text { enseñanza y el } \\
\text { aprendizaje }\end{array}$ & $\begin{array}{l}\text { Otorgan importancia } \\
\text { a la posibilidad de } \\
\text { acceso a la } \\
\text { computadora, } \\
\text { recurso del que } \\
\text { carecen. Así también, } \\
\text { reconocen el impacto } \\
\text { positivo en los } \\
\text { procesos de } \\
\text { socialización de los/ } \\
\text { as niños/as. Valoran } \\
\text { las NT como } \\
\text { facilitadoras }\end{array}$ & $\begin{array}{l}\text { Valoran } \\
\text { positivamente su } \\
\text { uso en tanto } \\
\text { promueven formas } \\
\text { renovadas de } \\
\text { enseñar y de } \\
\text { aprender. Para } \\
\text { ambos casos, las } \\
\text { docentes } \\
\text { entrevistadas, } \\
\text { sostienen que en } \\
\text { laEnseñanza, las } \\
\text { NT, ofrecen } \\
\text { recursos y }\end{array}$ & $\begin{array}{l}\text { La utilización de la } \\
\text { computadora y la } \\
\text { proyección de } \\
\text { películas en el cañón, } \\
\text { contribuyen de forma } \\
\text { positiva en el } \\
\text { desarrollo y } \\
\text { explicación de los } \\
\text { contenidos en las } \\
\text { clases. } \\
\text { La posibilidad de } \\
\text { "confrontar } \\
\text { materiales" digitales } \\
\text { y textos }\end{array}$ \\
\hline
\end{tabular}




\begin{tabular}{|c|c|c|c|}
\hline & $\begin{array}{l}\text { de un aprendizaje } \\
\text { participativo. }\end{array}$ & $\begin{array}{l}\text { herramientas que } \\
\text { "atrapa" el interés } \\
\text { en los/as niños/as, } \\
\text { se “enganchan } \\
\text { más". } \\
\text { Las docentes } \\
\text { interactúan entre lo } \\
\text { digital y el papel. } \\
\text { Sostienen que si } \\
\text { bien a los/as niños/ } \\
\text { as les "encanta" lo } \\
\text { digital, muchos } \\
\text { prefieren lo } \\
\text { concreto: trabajar } \\
\text { con el formato } \\
\text { papel. } \\
\text { En la interacción } \\
\text { con los niños/as, se } \\
\text { aprende } \\
\text { participativa y } \\
\text { colaborativamente. }\end{array}$ & $\begin{array}{l}\text { tradicionales fomenta } \\
\text { el debate para que } \\
\text { los/as estudiantes } \\
\text { asuman una postura } \\
\text { crítica respecto de los } \\
\text { mismos. El video, } \\
\text { aporta la posibilidad } \\
\text { de ver material } \\
\text { documental como } \\
\text { fuente de época, así } \\
\text { también, promueve } \\
\text { un aprendizaje visual } \\
\text { que mejora la } \\
\text { internalización de los } \\
\text { contenidos. }\end{array}$ \\
\hline $\begin{array}{l}\text { Finalidades } \\
\text { educativas } \\
\text { relacionadas } \\
\text { a las NT }\end{array}$ & $\begin{array}{l}\text { No se observan } \\
\text { claramente definidas, } \\
\text { en términos de } \\
\text { intenciones } \\
\text { planificadas. }\end{array}$ & $\begin{array}{l}\text { No se observan } \\
\text { claramente } \\
\text { definidas, en } \\
\text { términos de } \\
\text { intenciones } \\
\text { planificadas. }\end{array}$ & $\begin{array}{l}\text { No se observan } \\
\text { claramente definidas, } \\
\text { en términos de } \\
\text { intenciones } \\
\text { planificadas. }\end{array}$ \\
\hline Dificultades & $\begin{array}{l}\text { Manifiestan la } \\
\text { necesidad de } \\
\text { fortalecer la } \\
\text { formación específica. } \\
\text { Mencionan que el } \\
\text { desconocimiento les } \\
\text { exige dedicar mayor } \\
\text { tiempo del usual a la } \\
\text { planificación de } \\
\text { actividades. } \\
\text { Advierten que la } \\
\text { actualización de los } \\
\text { soportes es tan rápida }\end{array}$ & $\begin{array}{l}\text { Falta de formación } \\
\text { específica, de } \\
\text { conectividad que } \\
\text { permita a todos/as } \\
\text { las/os docente } \\
\text { acceder a los } \\
\text { tutoriales. No todos/ } \\
\text { as cuentan con una } \\
\text { PC. } \\
\text { Incompatibilidad de } \\
\text { software entre } \\
\text { Windows y Linux. } \\
\text { Esto imposibilita }\end{array}$ & $\begin{array}{l}\text { Falta de internet en la } \\
\text { escuela, como } \\
\text { también las fallas del } \\
\text { servidor intranet. } \\
\text { Visión crítica del } \\
\text { material que es } \\
\text { incluido y } \\
\text { promocionado a } \\
\text { través plan "conectar } \\
\text { igualdad", ya que si él } \\
\text { o la docente no tiene } \\
\text { un buen dominio y } \\
\text { flexibilidad en su uso, }\end{array}$ \\
\hline
\end{tabular}




\begin{tabular}{|c|c|c|c|}
\hline & $\begin{array}{l}\text { que quedan } \\
\text { obsoletos. } \\
\text { El deterioro de los } \\
\text { equipos ocasiona que } \\
\text { los/las docentes } \\
\text { deban "adaptar" o } \\
\text { "reemplazar" } \\
\text { actividades a fin de } \\
\text { poder llevar adelante } \\
\text { una secuencia } \\
\text { didáctica. } \\
\text { Deficiente el acceso a } \\
\text { internet para el } \\
\text { trabajo en red. }\end{array}$ & $\begin{array}{l}\text { trabajar en la casa, } \\
\text { dado que la mayoría } \\
\text { de las netbook } \\
\text { facilitadas por el } \\
\text { programa nacional, } \\
\text { poseen Linux. } \\
\text { Cambio permanente } \\
\text { y diversidad de } \\
\text { programas y } \\
\text { software que no } \\
\text { permiten asimilar } \\
\text { funciones para su } \\
\text { aplicación. }\end{array}$ & $\begin{array}{l}\text { los contenidos } \\
\text { poseen un esquema } \\
\text { muy fijo de "bajada } \\
\text { de línea". } \\
\text { Se significan como } \\
\text { "limitados" los } \\
\text { contenidos o } \\
\text { plantillas vinculados } \\
\text { a las ciencias sociales } \\
\text { e historia. } \\
\text { Claras limitaciones } \\
\text { de infraestructura en } \\
\text { el uso de "las } \\
\text { compus". Falta de } \\
\text { equidad. No todos los } \\
\text { estudiantes tienen } \\
\text { computadora y } \\
\text { muchas están } \\
\text { dañadas. Las mismas } \\
\text { tienen poca } \\
\text { capacidad de } \\
\text { memoria, lo que } \\
\text { imposibilita } \\
\text { acumular } \\
\text { información y } \\
\text { materiales. }\end{array}$ \\
\hline $\begin{array}{l}\text { Organización } \\
\text { de secuencias } \\
\text { didácticas }\end{array}$ & $\begin{array}{l}\text { Se encuentran } \\
\text { establecidos días y } \\
\text { horarios fijos para el } \\
\text { uso de NT. Las } \\
\text { secuencias didácticas } \\
\text { que se organizan } \\
\text { específicamente para } \\
\text { tal fin, por lo general } \\
\text { se articulan juegos y } \\
\text { o videos con los } \\
\text { contenidos } \\
\text { planificados. }\end{array}$ & $\begin{array}{l}\text { Se establecen } \\
\text { momentos claros y } \\
\text { concretos en las que } \\
\text { se incorporan las } \\
\text { NT en las clases. Se } \\
\text { presentan temas, } \\
\text { imágenes, videos y } \\
\text { se complementan } \\
\text { con otro soporte. }\end{array}$ & $\begin{array}{l}\text { Se establecen } \\
\text { momentos claros y } \\
\text { definidos. Por lo } \\
\text { general, el uso de NT } \\
\text { se vincula al cierre de } \\
\text { una secuencia } \\
\text { didáctica. Se hace } \\
\text { hincapié en el } \\
\text { desarrollo de los } \\
\text { conceptos para evitar } \\
\text { que se valoren las NT } \\
\text { desde una visión } \\
\text { exclusivamente } \\
\text { lúdica. }\end{array}$ \\
\hline
\end{tabular}


Historiadores/as y profesores/as no solo han debido afrontar los cambios disciplinares y las innovaciones epocales o las perspectivas teóricas metodológicas que han caracterizado el oficio, sino que también han tenido que familiarizarse con el impacto que provocó la revolución de las nuevas tecnologías de la información y comunicación, como es internet. Esta innovación tecnológica, plantea importantes desafíos para quienes trabajamos con la información, como es el caso de los/as historiadores/as, ya que nos impone una nueva forma de comunicación, interacción, de entender y ver la realidad, como así también de estudiarla. Se amplía el acceso y conocimiento de nuevas fuentes, se abren archivos, se conoce más sobre lo que acontece en otros espacios y se interviene de manera más rápida y eficaz sobre los "repositorios de memorias"

Los/as historiadores/as y profesores/as contamos historias y lo hacemos construyendo textos escritos. "La historia es hija de la era de la imprenta"; quizás por ello, la utilización de nuevas tecnologías ponga en tensión la forma tradicional de enseñar historia o trasmitir el conocimiento histórico. León Goldestein sostiene que "nada importante parece haber cambiado, hasta que irrumpieron las TIC, afectando de manera directa la manera como se genera el conocimiento y como se lo distribuye en la sociedad" (citado en Muñoz Delaunoy, 2013).

Los cambios en la construcción y comunicación del conocimiento, originados por las nuevas tecnologías, muestran los problemas prácticos que se originan a partir de su utilización. Pero debemos tener una visión positiva, considerar que la historia y los/as profesores/as que enseñamos historia encontraremos nuevas formas de transmitir el conocimiento, de forma tal que "encante a los estudiantes", utilizando las nuevas tecnologías de manera que sintonice con las expectativas de un público exigente y con las demandas de nuestra época. Ello presupone el desafío y una perspectiva superadora, en tanto que las nuevas tecnologías pueden ser soportes didácticos sumamente ricos, y que pueden contribuir a superar la apatía que genera la historia en muchos jóvenes.

Como sostiene Muñoz Delaunoy (2013, p.394) "Es necesario que los historiadores y los especialistas en Didáctica desempeñen un papel más activo con relación a los cambios. Deben ser ellos y no el mercado quienes definan en qué frente especifico vale la pena alentar innovaciones".

La escuela de hoy se debate entre la tradición y la innovación sobre el mandato social de reproducción cultural y transmisora de determinados 
conocimientos para la formación de ciudadanías. Las preocupaciones del qué y cómo enseñar se renuevan ante un para qué que se actualiza velozmente. Qué información, qué saberes, qué conocimiento nos ha llevado a la necesidad de construcción de currículos sobrecargados, por un lado y al inmovilismo e incertidumbre desesperante por el otro. La escuela, en este escenario, no ha podido aun plantearse nuevas preguntas a problemas diversos, sigue la tradición de dar respuestas, desde el conocimiento acabado y esto se presenta como un límite ante la vertiginosidad de los cambios, entendemos, que debería promoverse una mirada atenta para la construcción, siempre actualizada, de saberes que configuren racionalidades distintas.

Los/as estudiantes actuales, son la primera generación cien por ciento tecnológica, plenamente inmersa en los códigos de la era digital; y esto ya nos marca una importante diferencia con generaciones anteriores al boom digital. Como nativos de ese mundo, los/as jóvenes se comunican de una manera que los/as adultos/as no dominan. Es evidente la brecha que existe entre el lenguaje de éstos y el de los/as jóvenes. Quizás sea por ello que los/as docentes consideren que el lenguaje de los/as estudiantes es muy acotado, y a los estudiantes no entusiasme el utilizado por el docente.

Internet ha permitido que los/as adolescentes de hoy dispongan y accedan a una cantidad de información, que antes era inimaginable y este es un potencial que debemos adjudicar a la cultura digital. La red permite encontrar una cantidad enorme de recursos que permiten a docentes y estudiantes hacer y enseñar historia. La utilización de la red estimula la interacción y la comunicación en los procesos de enseñanza y aprendizaje.

Son muchos los disparadores que emanan de las entrevistas realizadas. Sin embargo, deberíamos seguir indagando en los alcances del formato digital en la clase de historia o ciencias sociales, para poder sistematizar experiencias y ofrecer propuestas que nos inviten a pensar en nuevos modos de construcción metodológica (Edelstein, 1996).

\section{NotAS PARA SEGUIR PENSANDO}

En primer lugar quisiéramos resaltar la importancia del cuestionario como técnica o estrategia en la investigación cualitativa critica, en la medida que nos ofrece un primer panorama general de los sentidos, usos, y conocimientos 
que el profesorado atribuye a las nuevas tecnologías en la enseñanza. Cuestión que anticipa posibles preguntas o núcleos de problemas para profundizar en las entrevistas.

Por otro lado existe, según podemos ver en los datos, diversidad de usos, accesos y conocimientos de las nuevas tecnologías. Igualmente distintos son los modos en que el profesorado ha ido incorporándola a sus prácticas de la enseñanza. Evidentemente no existe un desconocimiento y disponibilidad de los artefactos digitales en la vida de las personas, sin embargo no se termina de configurar como una nueva cultura escolar.

La diversidad de soportes y de infraestructuras existentes en las instituciones educativas, según la muestra, es significativa. Aunque debemos afirmar que son escasas las escuelas que han desarrollado proyectos pedagógicos-didácticos que promuevan la incorporación de estos recursos a la práctica de la enseñanza. Reconocen, los/as encuestados/as, la existencia de programas, proyectos y planes - tanto nacionales como jurisdiccionales - pero su implementación no se concreta en las escuelas. También, la ausencia de capacitación y formación específica que posibilite complejizar el uso de los recursos es un dato interesante para seguir indagando.

Finalmente, con el objetivo de profundizar la indagación, creemos que es oportuno recoger experiencias concretas y realizar observaciones participantes, de manera que nos permitan ampliar la mirada y comprender los criterios que orienta al profesorado cuando utiliza las tecnologías en la clase de historia o ciencias sociales. En este sentido, los artículos que siguen en este dossier, dan cuenta de algunas prácticas del profesorado. Esbozaremos algunas de esas experiencias en la enseñanza de la historia y/o ciencias sociales a partir de incorporar y utilizar la cultura digital en el aula.

\section{REFERENCIAS}

BISQUERRA, Rafael. Métodos de investigación educativa: guía práctica. Barcelona: CEAC, 2000.

DUSSEL, Inés. La escuela y los nuevos medios digitales. Notas para pensar las relaciones con el saber en la era digital. In: AA.VV. La Educación Alterada: aproximaciones a la escuela del siglo XXI. Córdoba: Universidad Nacional de Villa María (Eduvim); Salidas al mar ediciones, 2010. 
EDELSTEIN, Gloria. Un capítulo pendiente: el método en el debate didáctico contemporáneo. In: AAVV. Corrientes didácticas contemporáneas. Buenos Aires: Paidós, 1996.

FIDELI, Roberto; MARRADI, Alberto. Intervista. In: Enciclopedia delle scienze sociali. Roma: Treccani, 1996.

JARA, Miguel Angel; SANTISTEBAN, Antoni. Las representaciones de los estudiantes sobre la temporalidad y la HRP en la formación inicial del profesorado de historia. Aportes de una investigación. Reseñas de Enseñanza de la Historia, Córdoba: Alejandría, año 8, n.8, nov. 2010.

MARGULIS, Mario; URRESTI, Marcelo (2008). La juventud es más que una palabra. In: MARGULIS, Mario (Ed.) La juventud es más que una palabra: ensayos sobre cultura y juventud. Buenos Aires: Biblos, 2008.

MARRADI, Alberto; ARCHENTI, Nélida; PIOVANI, Juan Ignacio. Metodología de las Ciencias Sociales. Buenos Aires: Emecé, 2007.

MUÑOZ DELAUNOY, Ignacio. Enseñar historia en la "era digital". In: MUÑOZ DELAUNOY, Ignacio; OSSANDÓN MILLAVIL, Luis (Comp.) La didáctica de la historia y la formación de ciudadanos en el mundo actual. Santiago de Chile: Dibam, 2013.

WALKER, Rob. Métodos de investigación para el profesorado. Madrid: Morata, 1989.

\section{NOTAS}

${ }^{1}$ Un primer análisis lo hemos presentado en la ponencia "Los procesos de construcción de un instrumento para la investigación en didáctica de la historia. El cuestionario"; presentada en XV Jornadas Nacionales y IV Internacionales de Enseñanza de la Historia, organizadas por Apehun y la Facultad de Ciencias Humanas de la UNL, Santa fe, septiembre de 2014.

${ }^{2}$ El cuestionario se realizó en tres localidades de la provincia de Rio Negro (Cipolletti, San Antonio Oeste y San Carlos de Bariloche) y en la Ciudad de Neuquén capital. Ambas provincias ubicadas en la norpatagonia argentina. En cada una de las localidades se encuestó a docentes de tres instituciones educativas de cada nivel (inicial, primaria y media).

${ }^{3}$ Para profundizar una caracterización y análisis de las reformas y contrarreformas educativas para el nivel medio en las provincias de Río Negro y Neuquén, pueden consultarse: Muñoz, María E.; Salto, Víctor A. (2005). El proceso de reforma educativa en la Provincia de Neuquén: breve estado actual de situación. Córdoba: Reseñas de enseñanza de la historia, n.3. Apehun. Universitas (p.175-193); Jara, Miguel A. y Blanco, Laura S.; (2006). El lugar de la historia en los Diseños Curriculares de la Prov. de Río Negro. Entre reformas y contrarreformas. Córdoba: Reseñas de enseñanza de la historia, n.4, Apehun. Universitas 
(p.135-156) y Funes, Graciela (2013). Historias enseñadas recientes. Utopías y prácticas. Neuquén: Educo (p.179-272), entre otros.

${ }^{4}$ Decreto presidencial n. 459/2010.

${ }^{5}$ En tanto forma de conversación, la vida cotidiana y los procesos de socialización - como plantean Valles (1997) y Alonso (1998), entre otros - pueden constituirse en la base para la caracterización preliminar y el aprendizaje de la entrevista. En efecto, en la vida cotidiana estamos recurrentemente involucrados en prácticas conversacionales que implican el intercambio de información; las competencias para desempeñarnos en ellas, que se adquieren a través de la socialización, empezando por el lenguaje y las capacidades del habla, y siguiendo por el conjunto de saberes tácitos que hacen posibles tales interacciones verbales, son cruciales también en la práctica de la entrevista de investigación social (MARRADI; ARCHENTI; PIOVANI, 2007, p.216).

Artigo recebido em 8 de abril de 2016. Aprovado em 30 de maio de 2016. 